\title{
Transient Radio-frequency Ground Waves Over the Surface of a Finitely Conducting Plane Earth
}

\author{
J. R. Johler
}

\begin{abstract}
The complete transient signal is reconstructed after propagation via the ground-wave mode over a finitely conducting plane earth in which the displacement currents are neglected. The theory is illustrated by computations that have been made on formulas derived by the method of the inverse Laplace transformation. The results of this analysis indicate that current sources with sinusoidal form in the time domain could be used to simulate spheries. The methods employed in this analysis can be also used to reconstruct propagated signals of pulsed radio-navigation systems.
\end{abstract}

\section{Introduction}

In recent years considerable interest has developed in various types of electromagnetic ground-wave transients that propagate over the surface of the earth. This interest has in large measure been stimulated by the use of pulse techniques for precision radio-navigation systems and the ever presence of spheries that are propagated around the earth from various thunderstorms originating principally in the vicinity of the tropical land masses of the earth. In a previous paper by the author [1] ${ }^{1}$ and in papers by Wait $[2,3]$, a theory of propagation of the ground-wave transient was developed as an inverse Laplace transformation. The basic mathematical problem is the evaluation of the Fourier integral, which describes the transient signal, $E\left(t^{\prime}, d\right)$, in space and time as follows:

$$
E\left(t^{\prime}, d\right)=\frac{1}{2 \pi} \int_{-\infty}^{+\infty} \exp (i \omega t) f_{s}(\omega) \boldsymbol{E}(\omega, d) d \omega,
$$

where $f_{s}(\omega)$ is the source current transform, and $E(\omega, d)$ is the transfer characteristic of the propagation medium. The theory was illustrated [1] by an evaluation of the sinusoidal part of the signal. Such examples describe the complete signal at the larger values of time, at low frequencies, and over highly conducting earth. It was shown that a signal generated by a sinusoidal current source, which commences abruptly at some time, $t=0$, and which is allowed to continue oscillating indefinitely, reaches a steady state condition, the amplitude and phase of which correspond precisely to the continuous-wave type of signal, i. e., the signal not interrupted in time. It is the purpose of this paper to extend the theory and reconstruct the complete signal by the addition of certain nonsinusoidal terms, which appear as a result of the inverse Laplace transformation and which describe the early time behavior of the signal.

\section{Theory}

The transient signal, $E\left(t^{\prime}, d\right)$, may be represented over a finitely conducting plane earth in which the

\footnotetext{
1 Figures in brackets indicate the literature rcferences at the end of this paper.
}

displacement currents are negligible, for a vertically polarized source of the form, $\exp \left(-c_{1} t\right) \cos$ $\omega_{c} t(0<t<\infty)$, at the local time, $t^{\prime}$, as follows [1]:

$$
\begin{aligned}
E\left(t,^{\prime} d\right)= & \nu C \exp \left(-\nu t^{\prime}\right)\left\{-1-\nu \sqrt{\pi \alpha} \exp \left(\nu^{2} \alpha\right)\right. \\
& {\left.\left[\operatorname{erfc}(-\nu \sqrt{\alpha})-\operatorname{erfc}\left(\frac{t^{\prime}}{2 \sqrt{\alpha}}-\nu \sqrt{\alpha}\right)\right]+\frac{1}{a \nu}-\frac{1}{a^{2} \nu^{2}}\right\} } \\
& +\nu^{\prime} C \exp \left[\frac{-t^{\prime 2}}{4 \alpha}\right]+\nu C\left[\frac{1}{a^{2} \nu^{2}}\right]
\end{aligned}
$$

where

$$
\begin{aligned}
& \nu=c_{1}+i \omega_{c} \\
& \nu^{\prime}=\frac{t^{\prime}}{2 \alpha}+\nu \\
& C=2 \frac{I_{0} l a^{2}}{4 \pi \kappa d^{3}} \\
& \alpha=\frac{\eta_{1}^{3} d}{2 \sigma \mu_{0} c^{3}},
\end{aligned}
$$

and where

$d=$ the distance along the surface of the earth, in meters,

$C=2\left(10^{-7}\right) / d$, where $I_{0} l=1$ ampere-meter [4], $\eta_{1}=$ the index of refraction of air at the surface. $\eta_{1} \sim 1.000338$

$\sigma=$ the conductivity of the ground, in mhos/ meter,

$\mu_{0}=$ the permeability of space, $\mu_{0}=4 \pi \quad\left(10^{-7}\right)$ henry/meter,

$c=$ the speed of light. $\quad c \sim 2.997925\left(10^{8}\right)$ meters/ second,

$a=\left(\eta_{1} d\right) / c ; t-a=t^{\prime}$, the local time, and

$\rho_{1}=$ the numerical distance. $\rho_{1} \sim \alpha \omega^{2}$, neglecting displacement currents in the earth.

Also, the transient signal, $E^{\prime \prime}\left(t^{\prime}, d\right)$, for a source, exp $\left(-c_{1} t\right) \cos \omega_{c} t\left(0<t<T_{2}\right)$, i. e., a source interrupted at a time, $T_{2}$, may be represented as follows:

$$
E^{\prime \prime}\left(t^{\prime}, d\right)=E\left(t^{\prime}, d\right)-\exp \left(-\nu T_{2}\right) E\left(t^{\prime \prime}, d\right),
$$




$$
t^{\prime \prime}=t^{\prime}-T_{2} \quad\left(t^{\prime}>T_{2}\right)
$$

The continuous wave or the wave not interrupted in time is the special case: $t^{\prime}>>0, c_{1}=0, T_{2}=\infty$. The early values of time, $t^{\prime}>0$, in this case describe the development of the steady state.

The source function, $F_{s}(t)$, may be brought into existence less abruptly as follows:

$$
\begin{gathered}
F_{s}(t)=\exp (-\nu t)-\exp (-\xi t) \\
\xi=c_{2}+i \omega_{c} .
\end{gathered}
$$

$\nu$ has been previously described (2a), and $c_{2}$ is assigned a large positive value $\left(c_{2}>>c_{1}\right)$. The transform, $f(\omega),(1)$ may be written

$$
f(\omega)=f_{s}(\omega) E(\omega)
$$

or more generally

$$
f(\omega)=E(\omega)\left[f_{s, 1}(\omega)+f_{s, 2}(\omega)+\ldots .\right],
$$

where $f_{s, 1}(\omega), f_{s, 2}(\omega), \ldots$ are source functions that have been superposed to determine some complicated waveform. The solution of the space-time function, $E^{\prime}\left(t^{\prime}, d\right)$, for this source (6) then merely requires the sum of two waves calculated as previously described (2),

$$
E^{\prime}=E^{\prime}\left(t^{\prime}, d\right)=E_{\nu}\left(t^{\prime}, d\right)-E_{\xi}\left(t^{\prime}, d\right) .
$$

Figures 4, 5, and 6 illustrate this signal.

It is interesting to note the space-time function, $E\left(t^{\prime}, d\right)$, for the special case, $\omega_{c}=0$, or the case of a step function source, $u(t)$, exponentially damped, exp $\left(-c_{1} t\right) u(t),^{2}$

$$
\begin{array}{r}
E\left(t^{\prime}, d\right)=c_{1} C \exp \left(-c_{1} t^{\prime}\right)\left\{-1-c_{1} \sqrt{\pi \alpha} \exp \left(c_{1}^{2} \alpha\right)\right. \\
\left.\left[\operatorname{erfc}\left(-c_{1} \sqrt{\alpha}\right)-\operatorname{erfc}\left(\frac{t^{\prime}}{2 \alpha}-c_{1} \sqrt{\alpha}\right)\right]+\frac{1}{a c_{1}}-\frac{1}{a^{2} c_{1}^{2}}\right\} \\
+\left[\frac{t^{\prime}}{2 \alpha}+c_{1}\right] C \exp \left[\frac{-t^{\prime 2}}{4 \alpha}\right]+c_{1} C\left[\frac{1}{a^{2} c_{1}^{2}}\right]
\end{array}
$$

In this case, the imaginary part of the function, $E\left(t^{\prime}, d\right)$, vanished.

The source has been assigned the form $\exp \left(-c_{1} t\right)$ $\cos \omega_{c} t$ or without damping $\left(c_{1}=0\right), \cos \omega_{c} t$. The sine source, $\exp \left(-c_{1} t\right)$ sin $\omega_{c} t$ merely involves the formation of the product $-i E\left(t^{\prime}, d\right)$ or the real part becomes the positive imaginary $+\operatorname{Im} E\left(t^{\prime}, d\right)$. The sine source at zero frequency (11) therefore vanishes. It can be further concluded that the step function response of the ground-wave propagation medium for a step function current source, $u(t)$, is the particular case, $c_{1}=0$, or $\nu=0(2)$, which is simply,

$$
E\left(t^{\prime}, d\right)=\frac{t^{\prime}}{2 \alpha} C \exp \left[\frac{-t^{\prime 2}}{4 \alpha}\right]
$$

\section{Computation}

The detailed reconstruction of the complete transient signal over a finitely conducting plane earth, neglecting displacement currents in the earth, is shown in figs. 1 to 6 . The complete signal, $E=$ $E\left(t^{\prime}, d\right)(2)$, is presented as three terms,

$$
E=E\left(t^{\prime}, d\right)=E_{0}\left(t^{\prime}, \mathrm{d}\right)+E_{1}\left(t^{\prime}, d\right)+E_{2}\left(t^{\prime}, d\right) .
$$

The first term, $E_{0}=E_{0}\left(t^{\prime}, d\right)$, is called the sinusoid because it is multiplied by the time harmonic function $\exp \left(-\nu t^{\prime}\right)(2)$. The remaining terms are

$$
\begin{aligned}
& E_{1}=E_{1}\left(t^{\prime}, d\right)=\nu C\left\{\exp \left[\frac{-t^{\prime 2}}{4 \alpha}\right]+\frac{1}{a^{2} \nu^{2}}\right\} \\
& E_{2}=E_{2}\left(t^{\prime}, d\right)=\frac{t^{\prime}}{2 \alpha} C \exp \left[\frac{-t^{\prime 2}}{4 \alpha}\right]
\end{aligned}
$$

$E^{\prime}=E^{\prime}\left(t^{\prime}, d\right)$, (figs. $\left.4,5,6\right)$ describe a signal the source of which has been brought into existance less abruptly (6).

Since the complete signal is very complicated, this presentation (13) illustrates the influence of the various parts of the signal as space-time and frequency parameters are varied. Thus, for example, the term $E_{2}$ degenerates into a "spike" close to the origin of time, $t^{\prime}$, as the frequency is decreased (fig. 6 ), and vanishes for the less abrupt source (6) such that the terms $E_{0}+E_{1}$ suffice to describe the complete signal. This is consistent with the limiting condition of very small numerical distance $(\sigma=\infty)$ or an attenuation factor of unity. Thus, the transform of the Norton surface wave at considerable distance from the source may be written

$$
E(s)=1 \quad(\nu=0, \alpha=0),
$$

and the space-time function, $E\left(t^{\prime}, d\right)$, is

$$
E\left(t^{\prime}, d\right)=\mathscr{L}^{-1} E(s)=\delta\left(t^{\prime}\right),
$$

where $\delta\left(t^{\prime}\right)$ is the unit impulse function at the origin of time. Therefore, the transition to infinite conductivity illustrates the development of the Dirac impulse function, $\delta\left(t^{\prime}\right)$.

The reconstruction of a radio-navigation type of pulse is illustrated (fig. 7) with the "classical" sinesquared current source. The transient is allowed to reach the steady state because the "leading edge" of the pulse is of primary interest. It is common practice to interrupt radio-navigation pulses at a time, $T_{2},(4,5)$ or apply exponential damping after some time, $T_{2}$, has elapsed. The source is redefined as follows:

$$
\begin{gathered}
\operatorname{Re} F_{s}(t)=\sin ^{2} \omega_{p} t \sin \omega_{c} t \quad(0<t<\infty), \\
F_{s}(t)=\frac{i}{2} \exp (-\nu t)-\frac{i}{4} \exp \left(-\nu_{1} t\right)-\frac{i}{4} \exp \left(-\nu_{2} t\right),
\end{gathered}
$$



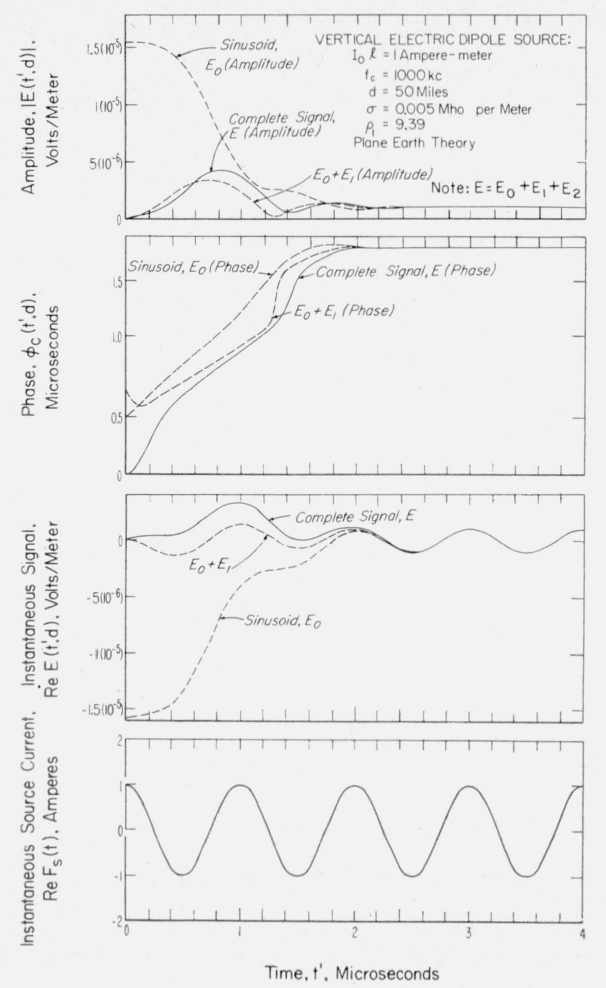

Figure 1. Amplitude, phase, instantaneous signal, and corresponding source current for propagated surface-wave transient.
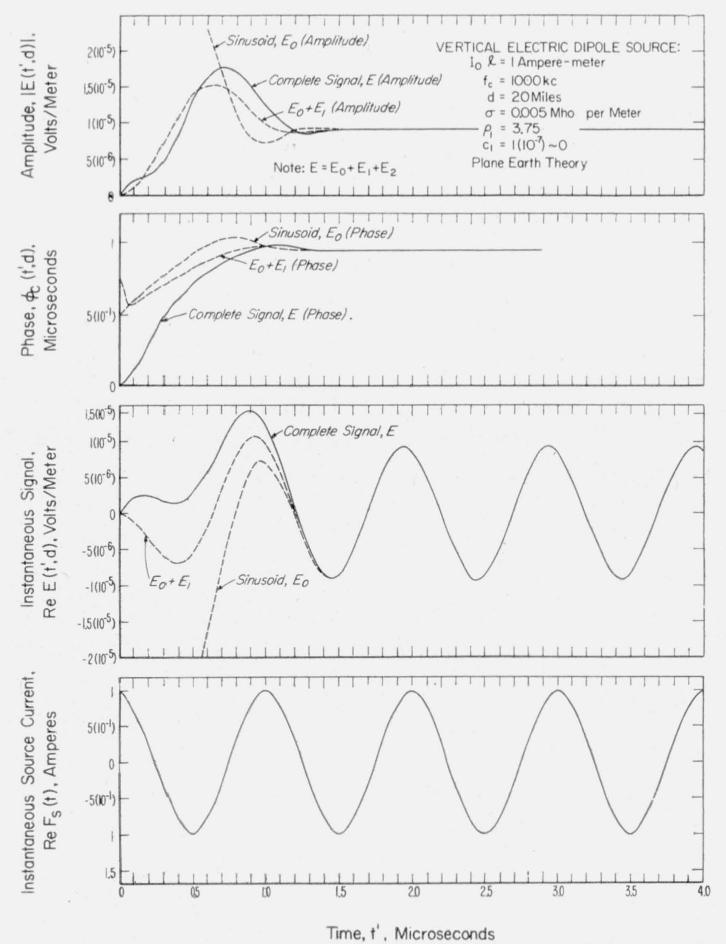

Figure 2. Amplitude, phase, instantaneous signal, and corresponding source current for propagated surface-wave transient.
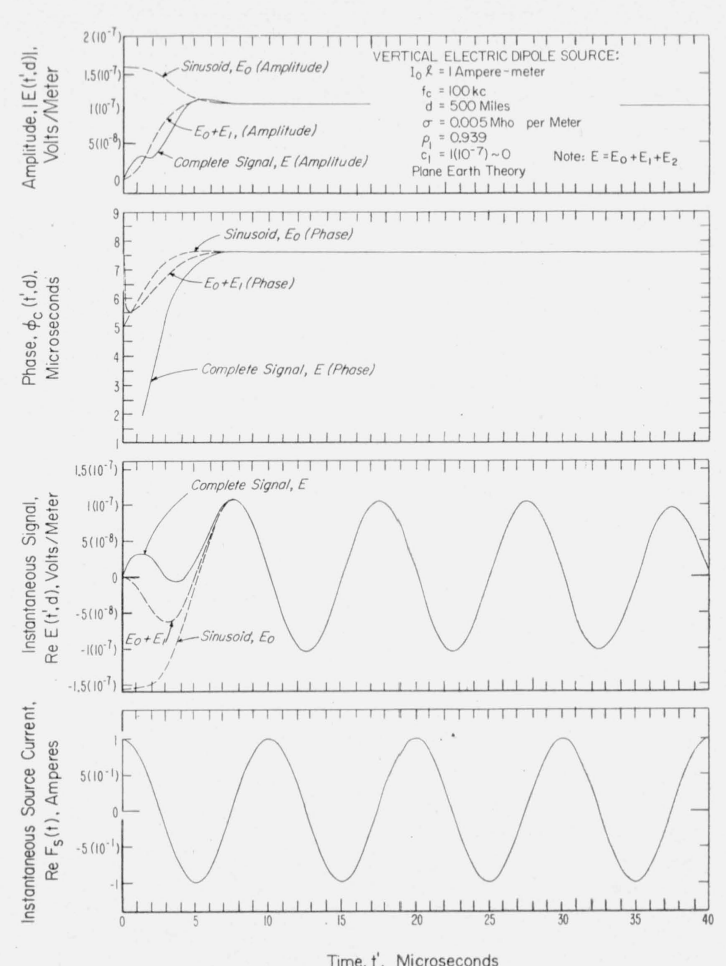

Figure 3. Amplitude, phase, instantaneous signal, and corresponding source current for propagated surface-wave transient.
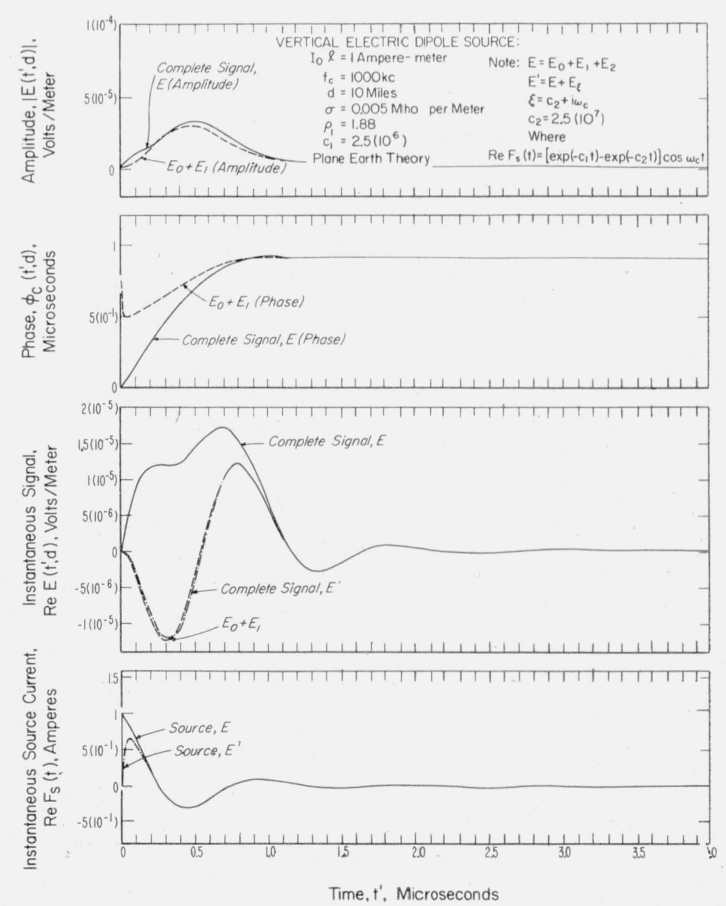

Figure 4. Amplitude, phase, instantaneous signal, and corresponding source current for propagated surface-wave transient. 

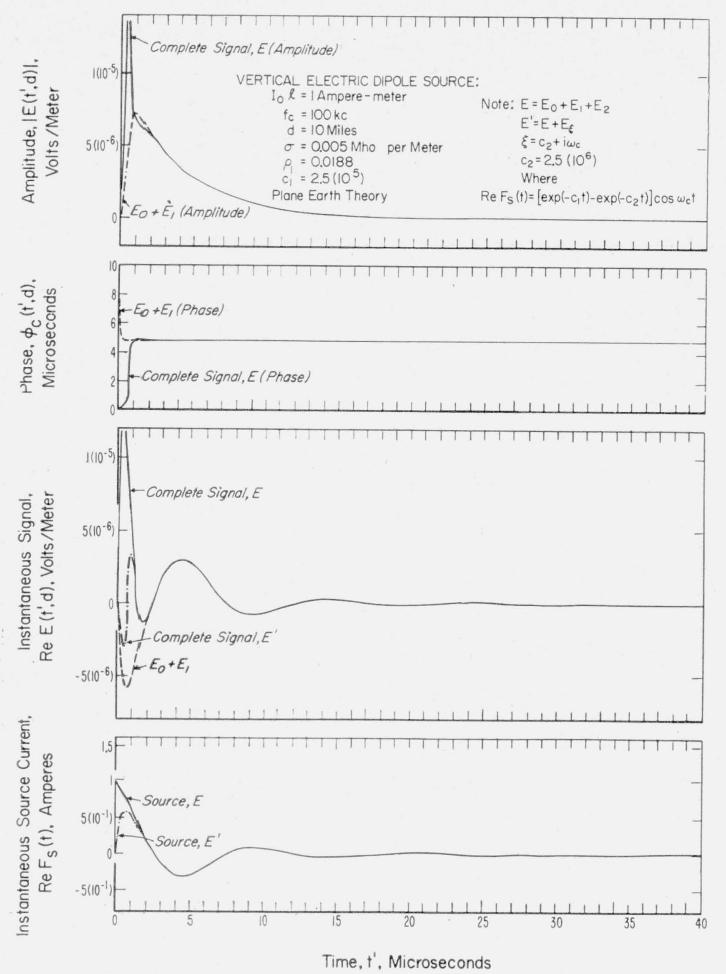

Figure 5. Amplitude, phase, instantaneous signal, and corresponding source current for propagated surface-wave transient.
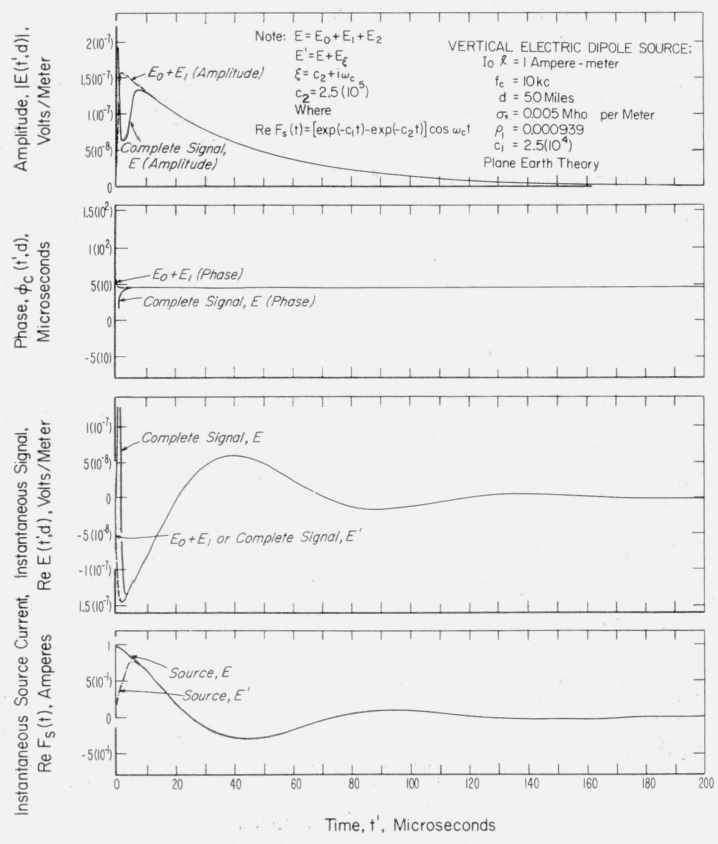

Figure 6. Amplitude, phase, instantaneous signal, and corresponding source current for propagated surface-wave transient.
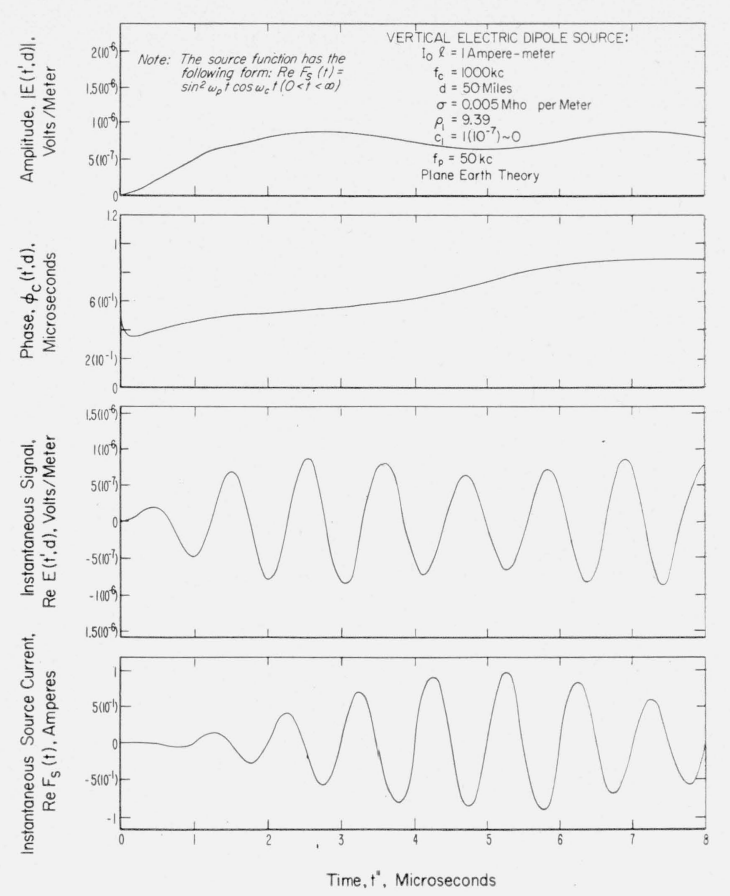

FIgure 7. Amplitude, phase, instantaneous signal, and corresponding sine squared source current for propagated surfacewave transient.

where

$$
\begin{aligned}
\nu & =c_{1}+i \omega_{c} \text { (as before), } \\
\nu_{1} & =c_{1}+i\left(\omega_{c}+2 \omega_{p}\right), \\
\nu_{2} & =c_{1}+i\left(\omega_{c}-2 \omega_{p}\right) .
\end{aligned}
$$

The exponential damping factor, $c_{1}$, may be assigned a finite value or zero. The space-time function, $E\left(t^{\prime}, d\right)$, may therefore be written as the sum of three oscillatory waves;

$$
E\left(t^{\prime}, d\right)=\frac{i}{2} E_{\nu}\left(t^{\prime}, d\right)-\frac{i}{4} E_{\nu 1}\left(t^{\prime}, d\right)-\frac{i}{4} E_{\nu 2}\left(t^{\prime}, d\right) .
$$

Figure 7 describes the propagated wave (20). Each term is calculated as described previously (2) $\left(c_{1}=0\right)$. The dispersion of the pulse at early times is a result of the transient behavior of the ground wave. The dispersion of the pulse at later times is a result of the operation of the propagation medium on the widely separated frequency spectra that develop after considerable time, $t^{\prime}$, has elapsed. It is interesting to suggest that the amplitude and phase could simulate the output of amplitude and phase envelope detectors of a radio receiver. However, the finite bandwidth of the receiver, $f_{\tau}(\omega)$, should be introduced into the transform, $f(\omega),(9)$ as follows:

$$
\begin{array}{r}
f(\omega)=E(\omega)\left[f_{s, 1}(\omega)+f_{s, 2}(\omega)+\ldots\right] \\
{\left[f_{r, 1}(\omega) f_{r, 2}(\omega) \ldots\right],}
\end{array}
$$

where $f_{r, 1}(\omega), f_{\tau, 2}(\omega), \ldots$ are the transfer characteristics of the frequency selective networks in the receiver circuit. 


\section{Conclusion}

The propagated transient signal over a finitely conducting plane earth can be reconstructed in detail, for current sources of complicated form, for all values of local time, by application of the inverse Laplace transformation. The most important merit of this analysis is the insight gained into the details of the propagation mechanism. It should be emphasized that the reconstruction of the complete signals at times less than approximately 0.1 microsecond requires the introduction of displacement currents and earth curvature, which have been neglected in this paper [3]. The method does however serve as a check on the tedious numerical methods of integration of the Fourier integral, the direct evaluation of which seems to be the obvious method of introducing into the transient solution of the ground wave the effect of the earth curvature and the effect of the displacement currents in the earth.
The mathematical formulas were programed for the electronic computer by L. C. Walters, and the computations were performed on the electronic computer by C. M. Lilley, both of the National Bureau of Standards staff. The work was sponsored by the Air Force Cambridge Research Center, Cambridge, Mass., and the Radio Navigation project of the National Bureau of Standards, Boulder, Colo.

\section{References}

[1] J. R. Johler, Propagation of the radiofrequency ground wave transient over a finitely conducting plane earth, Geofis. pura e appl. 37 (May-August 1957).

[2] J. R. Wait, Transient fields of a vertical dipole over a homogeneous curved ground, Can. J. Phys. 34, 27-35 (Jan. 1956)

[3] J. R. Wait, The transient behavior of the electromagnetic ground wave over a spherical earth, Trans. Inst. Radio Engrs. AP-5, No. 2 (April 1957).

[4] J. R. Johler, W. J. Kellar, and L. C. Walters, Phase of the low radiofrequency ground wave, NBS Circ. 573 (June $27,1956)$.

Boulder, Colo., August 30, 1957. 